\title{
Topological phase transitions driven by strain in monolayer tellurium
}

\author{
Wei Zhang, ${ }^{1,2,3}$ QuanSheng Wu, ${ }^{4,5}$ Oleg V. Yazyev,,${ }^{4,5}$ Hongming Weng, ${ }^{6,7, *}$ Zhengxiao Guo, \\ Wen-Dan Cheng, ${ }^{2}$ and Guo-Liang Chai ${ }^{2}$, \\ ${ }^{1}$ Fujian Provincial Key Laboratory of Quantum Manipulation and New Energy Materials, College of Physics and Energy, \\ Fujian Normal University, Fuzhou 350117, China \\ ${ }^{2}$ State Key Laboratory of Structural Chemistry, Fujian Institute of Research on the Structure of Matter, Chinese Academy of Sciences, \\ Fuzhou, Fujian 350002, China \\ ${ }^{3}$ Fujian Provincial Collaborative Innovation Center for Optoelectronic Semiconductors and Efficient Devices, Xiamen 361005, China \\ ${ }^{4}$ Institute of Physics, Ecole Polytechnique Fédérale de Lausanne, CH-1015 Lausanne, Switzerland \\ ${ }^{5}$ National Center for Computational Design and Discovery of Novel Materials MARVEL, Ecole Polytechnique Fédérale de Lausanne, \\ CH-1015 Lausanne, Switzerland \\ ${ }^{6}$ Beijing National Laboratory for Condensed Matter Physics and Institute of Physics, Chinese Academy of Sciences, Beijing 100190, China \\ ${ }^{7}$ Collaborative Innovation Center of Quantum Matter, Beijing 100084, China \\ ${ }^{8}$ Department of Chemistry, University College London, London WC1H OAJ, England, United Kingdom
}

(Received 3 June 2018; published 6 September 2018)

\begin{abstract}
Two-dimensional (2D) Xenes of a single type of element can offer fascinating electronic properties, such as massless Dirac fermions for extremely high charge-carrier mobility and topological insulators for dissipationless electron transport. However, the realization of either the massless Dirac fermions or the topological insulator in a same element system via a simple physical method has rarely been reported, which is of great importance for the development of next-generation electronic devices. Here, by using first-principles calculations, we identify that a 2D square tellurium system can be effectively tuned to realize either the massless Dirac fermions or the topological insulator phase. The 2D square tellurium system shows three structural phases via strain effect, i.e., buckled square, buckled rectangular, and planar square phases, which exhibit extraordinary topological properties. There are four anisotropic Dirac points in the buckled square phase, in which the Fermi velocity can be as high as $9.44 \times 10^{5} \mathrm{~m} / \mathrm{s}$. The buckled rectangular phase can behave as a quantum spin Hall insulator with a band gap of $0.24 \mathrm{eV}$, pointing towards promising applications for room-temperature devices. There also exist nodal lines in buckled square/planar square structures in the non-spin-orbit-coupling case. These findings extend the knowledge on single-layer materials and promote future applications of the $2 \mathrm{D}$ tellurium systems.
\end{abstract}

DOI: 10.1103/PhysRevB.98.115411

\section{INTRODUCTION}

The exceptional physical properties of graphene, such as the extremely high charge-carrier mobility which is mainly due to massless Dirac fermions [1], have triggered a flurry of exploration of two-dimensional (2D) materials hosting Dirac fermion quasiparticles [2-4] and their innovative applications in electronics, energy, and healthcare [5-7]. Especially, 2D materials of a single element $(X)$ have gradually become a new focus for realizing Dirac cones that show intriguing electronic transport properties for high-speed low-dissipation technologies $[8,9]$. In addition, they are also interesting for realizing topological insulators (TIs), which host quantum spin Hall effect (QSHE) along one-dimensional helical edge states, providing a new mechanism to realize nondissipative electronic transport for low-power multifunctional devices [10]. Till now, several 2D crystalline structures composed of a single element ranging from group III to group V were proposed, such as silicene, germanene, stanene, borophene,

\footnotetext{
*hmweng@iphy.ac.cn

${ }^{\dagger}$ g.chai@fjirsm.ac.cn
}

and phosphorene, where $X=\mathrm{Si}, \mathrm{Ge}, \mathrm{Sn}, \mathrm{B}$, and $\mathrm{P}$, respectively [11-14]. However, to the best of our knowledge, 2D Xenes composed of group VI elements are still poorly investigated and their properties need further investigation.

The existing 2D Xenes mainly show the honeycomb lattice, and the corresponding massless Dirac fermions are protected by the hexagonal symmetry. However, systems with other symmetries can also protect Dirac cone band dispersions $[15,16]$. For instance, square $\mathrm{MoS}_{2}$ [17], $\operatorname{Sn} X_{2}(X=\mathrm{S}, \mathrm{Se}$, or Te) with a square lattice [18] and Pmmn boron with rectangular lattices [19] possess Dirac bands. In addition, band overlap and a proper Fermi level are required to isolate the Dirac cone from other energy bands. Because of such strict requirements, systems with anisotropic Dirac cones are very exotic $[9,19]$. The discovery of new $2 \mathrm{D}$ materials with anisotropic Dirac cones will not only enrich the knowledge of Dirac physics but also widen the field of potential applications.

Here, by means of first-principles calculations, we identify that 2D square lattice tellurium is a versatile material with tunable anisotropic Dirac cones in which topological phase transitions can be realized via strain engineering. It shows three phases under different strain levels, i.e., buckled square (BS) phase, buckled rectangular (BR) phase, and planar square (PS) 
(a)

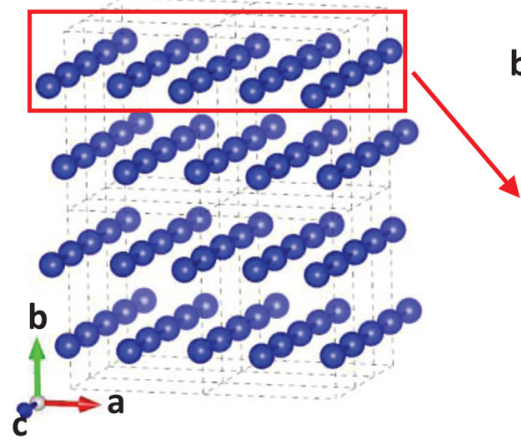

(d)

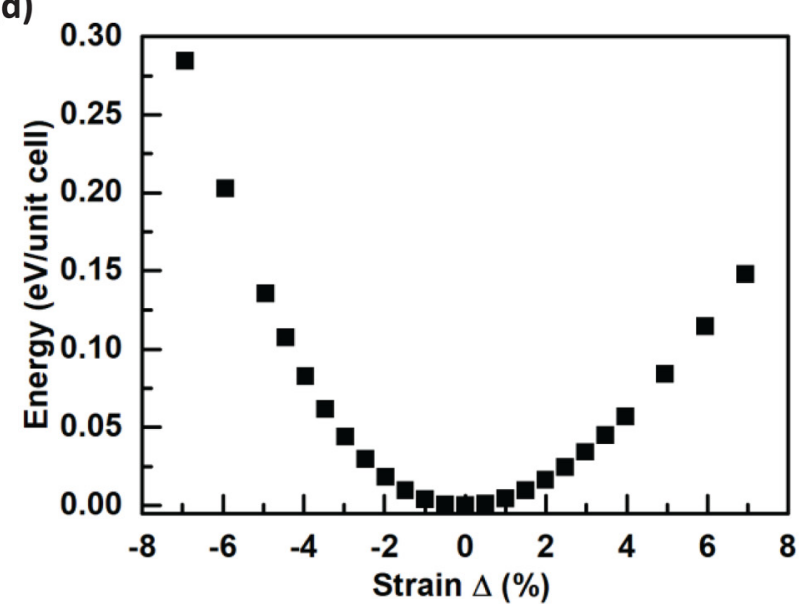

(b)

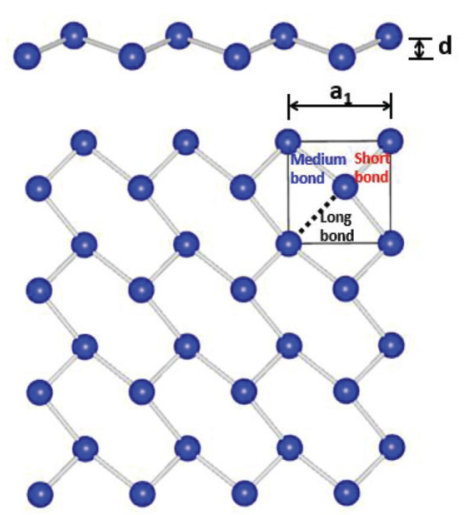

(c)
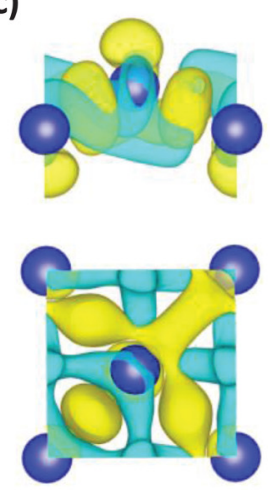

(e)

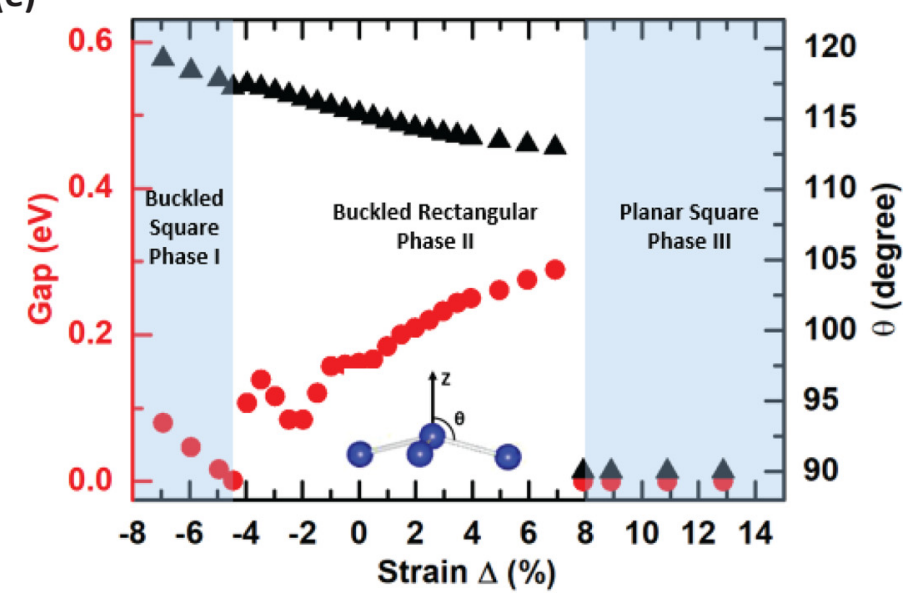

FIG. 1. 2D square tellurium system: (a) the tellurium monolayer cut from the experimental bulk structure. (b) Side and top views of the optimized structure. (c) Side and top views of the charge difference density of the system with an isosurface value of 0.0022 electrons $/ \AA^{3}$. Blue and yellow colors represent negative and positive electron density difference, respectively. (d) Energy variation vs applied strain. (e) Variations of the direct band gap at the Dirac points and the angle $\theta$ vs equiaxial strain in a series of structures. Red circles and black triangles mark the energy gap and angle $\theta$, respectively. The angle $\theta$ between the Te-Te bond and the direction normal to the plane is defined for clarity.

phase. In the non-spin-orbit-coupling (SOC) case, there exist nodal lines in the band structures of BS and PS phases. When SOC interaction is taken into account, the BS phase of $2 \mathrm{D}$ tellurium realizes a band structure with four anisotropic Dirac points, implying direction-dependent electronic transport, and the band dispersions can be described by a simple $k \bullet p$ model. The highest observed Fermi velocity of the Dirac points is $9.44 \times 10^{5} \mathrm{~m} / \mathrm{s}$, comparable to that of graphene $\left(10^{6} \mathrm{~m} / \mathrm{s}\right)$ [20]. Moreover, in the BR phase, the largest band gap and direct band gap for 2D quantum spin Hall insulators (QSHIs) are 0.29 and $0.24 \mathrm{eV}$, respectively. This is comparable to the largest band gap for TIs reported in previous studies [21-23]. These studies pave the way to applications of Dirac fermion systems and QSHIs in electronic and spintronic devices, and the exploration of new material candidates for realizing the quantum anomalous Hall effect [24].

\section{COMPUTATIONAL METHODS}

The first-principles calculations within density functional theory (DFT) were performed using the Vienna $a b$ initio simulation package $[25,26]$. The exchange-correlation energy was treated within the generalized gradient approximation in the form of Perdew-Burke-Ernzerhof [27]. The vacuum region in the supercell was set to about $20 \AA$ in order to avoid spurious interactions between the monolayer and its periodic images. The electron-ion interactions were treated using projector augmented wave potentials. Cutoff energy for plane-wave expansion was $400 \mathrm{eV}$. K-point meshes of $12 \times 12 \times 1$ and $24 \times 24 \times 1$ are employed for geometry optimization and self-consistent electronic structure calculations, respectively. Gaussian method is used for smearing with a parameter of $0.01 \mathrm{eV}$. Geometries were fully relaxed until the residual forces on each atom were less than $0.001 \mathrm{eV} / \AA$. The value of the band gap was verified using the nonlocal Heyd-Scuseria-Ernzerhof (HSE06) hybrid functional calculation $[28,29]$. The maximally localized Wannier functions [30,31] for Te $p$ orbitals were generated as the basis set and a tight-binding model was constructed to calculate $Z_{2}$ invariant and edge states for the system. For the interface structure of $\mathrm{BS} \mathrm{Te} / \mathrm{SrTiO}_{3}$, the bottom Te atom was assumed to be directly above the surface $\mathrm{Sr}$ atom of the $\mathrm{SrTiO}_{3}$ substrate at a distance of $\sim 3 \AA$. The van der Waals interaction within DFT $[32,33]$ was also considered to deal with interaction between monolayer tellurium and $\mathrm{SrTiO}_{3}$ for structure optimization [34]. 
(a) Buckled Square Phase I: $\Delta=-4.5 \%$

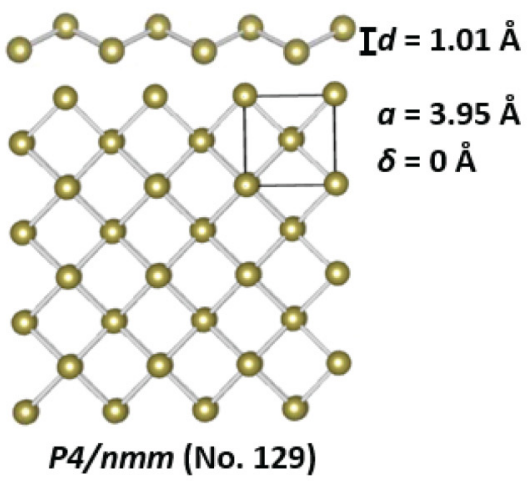

(b) Buckled Rectangular Phase II: $\Delta=\mathbf{4} \%$

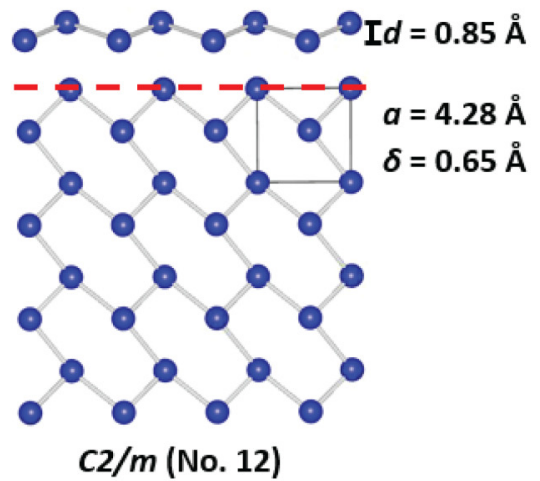

(c) Planar Square Phase III: $\Delta=13 \%$

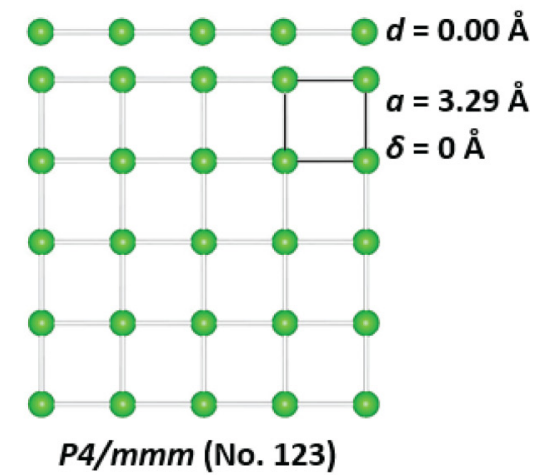

(d)

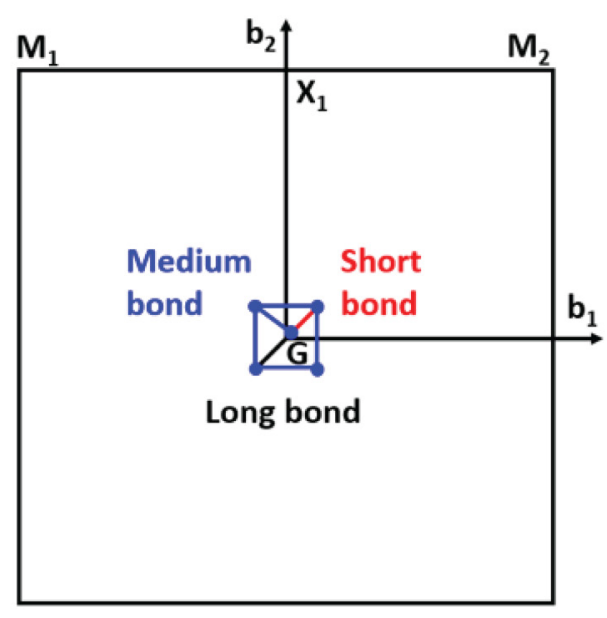

(e)

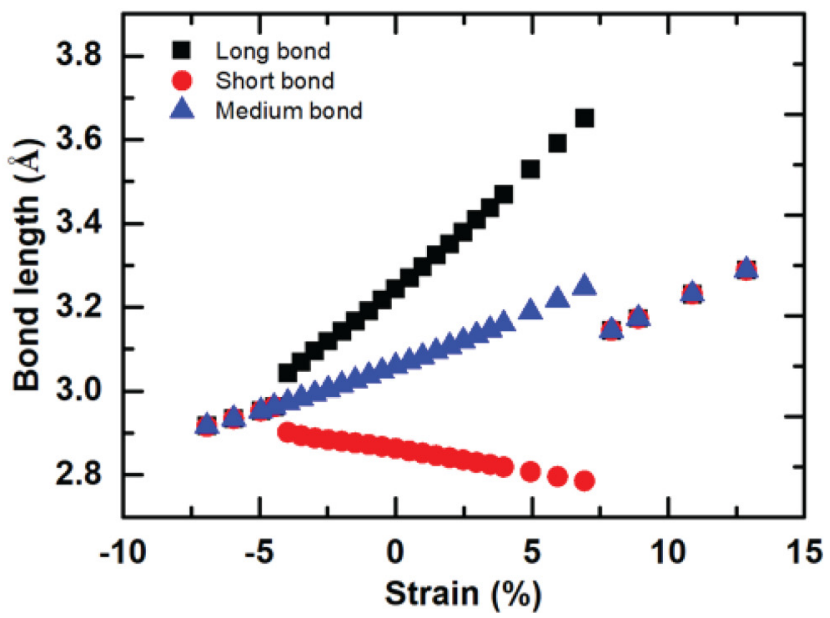

FIG. 2. Crystal structures and bond length variations vs applied strain for different phases of 2D square tellurium. For each phase, strain $\Delta$, thickness $d$, lattice constant $a$, and bond length difference $\delta$ between long and short bonds are also given. Supercells in side and top views for (a) structure with $\Delta=-4.5 \%$ in BS phase I, (b) structure with $\Delta=4 \%$ in BR phase II, and (c) structure with $\Delta=13 \%$ in PS phase III. (d) Definitions of the special point in the Brillouin zone with the real space lattice are given for reference. Three types of bond lengths are displayed, i.e., short, medium, and long bond lengths in each 2D tellurium structure. (e) Bond length variations vs applied strain effect.

\section{RESULTS AND DISCUSSION}

\section{A. Structures and topological phase transitions of the monolayer tellurium system}

Recent studies indicate that low-dimensional tellurium can show different structures which may lead to versatile electronic properties $[35,36]$. Previous studies also indicated that several materials based on a square or rectangular lattice can host the quantum spin Hall phase [17,37-39]. To date, the existing 2D materials can be mechanically exfoliated from their layered bulk counterparts or be grown epitaxially on proper substrates. Here, we found that a type of 2D tellurium system can be obtained by cutting from a corresponding experimental bulked structure [see Fig. 1(a)] [40]. The optimized structure is a 2D square tellurium as shown in Fig. 1(b), the electronic properties of which can be tuned by strain to present rich topological physics. For the 2D square tellurium, there are two inequivalent atoms per unit cell and three types of bonds, due to the central Te atom being displaced towards one corner of the unit cell. Its typical structure is a buckled structure with the optimized lattice constant $a_{0}=4.12 \AA$ and a band gap of $0.16 \mathrm{eV}$ [37]. Each Te atom forms three covalent bonds with the nearest Te atoms, as shown in Fig. 1(b). The remaining two electrons in the $p$ orbitals form a lone electron pair on each $\mathrm{Te}$ atom, which makes half of the Te atoms displace up and the other half displace down, relative to the plane of the material, thus forming a buckled structure. This is supported by the calculated charge difference density, as shown in Fig. 1(c). However, we found that the buckled structure can be tuned by strain engineering, as indicated by the calculated energy change versus strain in Fig. 1(d). Equiaxial strain $\Delta$ here is defined as $\left(a-a_{0}\right) / a_{0} \times 100 \%$ (positive strain refers to expansion, while negative strain refers to compression). We can see from Fig. 1(d) that the energy change is about $150 \mathrm{meV}$ when equiaxial strain $\Delta$ changes from -5 to $7 \%$, inducing a phase transition. The angle $\theta$ between the $z$ axis and the nearest-neighbor Te-Te bond was also calculated, as shown as the inset of Fig. 1(e).

Three phases of the 2D tellurium system can be distinguished according to the equiaxial strain applied. When equiaxial strain is applied with $\Delta \leqslant-4.5 \%$, the tellurium layer transforms into the BS phase I. The buckled crystal structure belongs to space group $P 4 / \mathrm{nmm}$ (no. 129) and one of two Te atoms is located at the center with coordinate $(0.5,0.5)$, shown 
in Fig. 2(a). Then, as noted from Fig. 1(e), over a large strain range from -4 to $7 \%$, the $2 \mathrm{D}$ tellurium monolayer remains stable as in the BR phase II ( $C 2 / m$, no. 12), and the Te atom in the middle moves off the center. Thus, the two Te atoms become inequivalent displayed in a bucked square lattice [see Fig. 2(b)]. Finally, when strain $\Delta$ increases to $8 \%$, the tellurium is pulled into the planar square structure $[P 4 / \mathrm{mmm}$, no. 123 , shown in Fig. 2(c)] and the system turns into PS phase III.

To understand the mechanism of the phase transition, we investigate in detail the strain dependence of bond lengths. There are three types of bonds in the unit cell for the square tellurium system: short bonds, long bonds, and medium bonds defined by different distances between the neighboring $\mathrm{Te}$ atoms, as illustrated in Fig. 2(d). It can be seen that two Te atoms locate themselves in the blue square real-space lattice and are represented as blue points. To see clearly the direction of each bond, we also provide the reciprocal-space lattice corresponding to the real-space lattice, illustrated as a black square in Fig. 2(d). Because the central Te atom displaces towards one corner of the square lattice, the long or short bond is created along the $\mathrm{M}_{2}-\mathrm{G}$ direction in reciprocal space and the medium bond is along the $\mathrm{M}_{1}-\mathrm{G}$ direction, which is perpendicular to the $\mathrm{M}_{2}-\mathrm{G}$ direction. Figure 2(e) displays the strain dependence of each bond length. In the BR phase II, the long or medium bond length increases with increasing the strain, while the short bond length decreases. When the system transforms into the BS phase I, the three bond lengths become equal and decrease with the decrease of strain. Similarly, at the transition point from the BR phase II to the PS phase III, the three bond lengths also become equal and increase with the increase of strain. This indicates that these two phase transitions are of the first order. The three phase transitions are characterized by different electronic properties associated with different topological features in the 2D tellurium system. The detailed results for these three phases are discussed as follows.

\section{B. Topological properties of the monolayer tellurium system}

First, the topological properties of phase I are investigated. The BS structures possess a nodal line (parallel to the $x y$ plane) when SOC is neglected, but transform into a 2D TI when considering SOC. The band structure without considering the SOC interaction is shown in Fig. 3(a). The valence and conduction bands near the Fermi level belong to different irreducible representations (IRs) of the $\mathrm{C}_{2 \mathrm{v}}$ point group: the IR of the band shown in red is $A_{1}$, while that shown in black is $B_{1}$ [see inset of Fig. 3(a)] [41]. Thus, band intersection can form a nodal line in the non-SOC case, protected by the PT (product of $\mathrm{P}$ and T) symmetry, as shown in Fig. 4(a). If the SOC is considered, the gap will be opened along the nodal line, leaving four Dirac point degeneracies.

At the phase transition point $(\Delta=-4.5 \%)$, the massless Dirac fermion bands remain intact. The calculated band structures with and without SOC for this BS structure $(\Delta=-4.5 \%)$ are compared in Figs. 3(a) and 3(b). Two linear band crossings (91 meV above the Fermi level) appear in the Brillouin zone (BZ) (along the $\mathrm{M}_{1}-\mathrm{G}$ and $\mathrm{G}-\mathrm{M}_{2}$ directions, respectively), which indicates the existence of Dirac cones with holes as charge carriers in this structure. This is further confirmed by the hybrid DFT calculations with the HSE06 functional. In fact, there are four Dirac points in this BS structure with
Buckled Square Phase I: $\Delta=-4.5 \%$
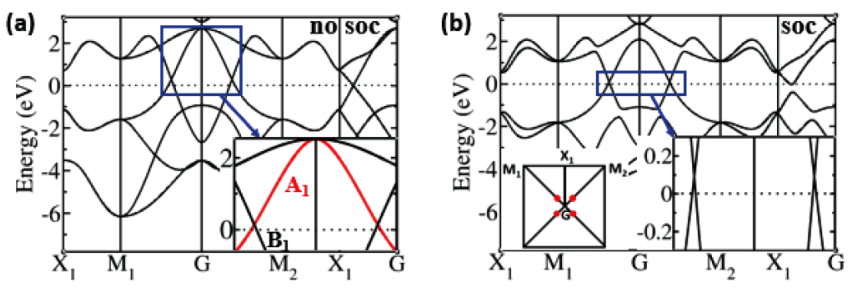

Buckled Rectangular Phase II: $\Delta=4 \%$
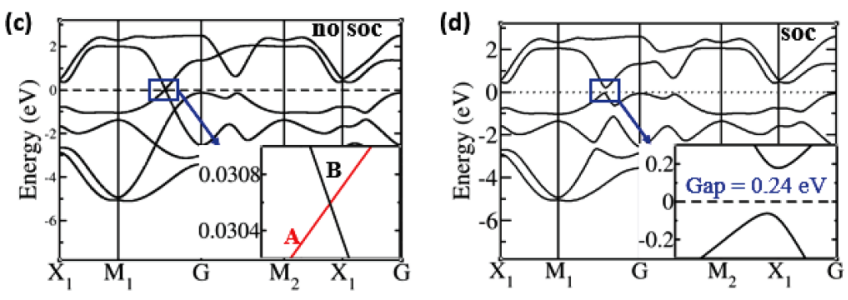

Planar Square Phase III: $\Delta=13 \%$
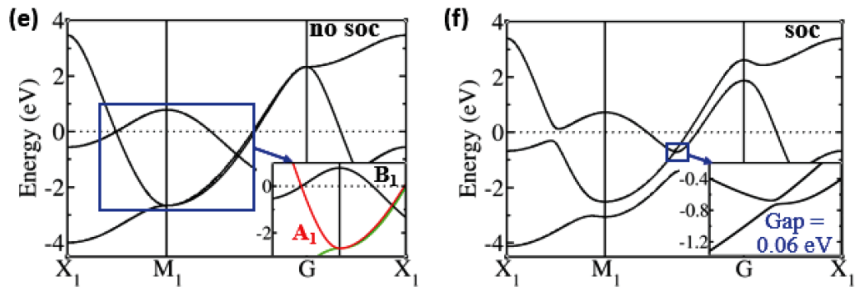

FIG. 3. Band structures for different phases of 2D tellurium. (a) Non-SOC and (b) SOC energy bands for the BS phase I ( $\Delta=-4.5 \%)$. The first Brillouin zone of the structure is shown in the inset. The locations of four Dirac points are indicated by red points. (c) NonSOC and (d) SOC energy bands for the BR phase II $(\Delta=4 \%)$. (e) Non-SOC and (f) SOC energy bands for the PS phase III $(\Delta=13 \%)$.

coordinates $( \pm 0.186, \pm 0.186)$, which are equivalent due to the fourfold rotation symmetry and illustrated as red dots in the BZ sketch in Fig. 3(b). When the strain further decreases $(\Delta<-4.5 \%)$, the band gap of the BS structure is opened at the Dirac points with a much larger value than that of graphene ( $\sim \mu \mathrm{eV})$ [42], as shown in Fig. 1(e). The SOC energy bands for BS structures with different strain are also shown in Fig. S1 of the Supplemental Material [43]. In the case of graphene, the band gap is due to the second-order contribution of SOC from $\pi$ band electrons, while the first-order SOC term has no contribution due to the flat geometry. In the case of considered BS configuration, the first-order SOC contribution is present because the hybridization between the $\pi$ and $\sigma$ orbitals is enabled in the highly buckled structure [4]. The Fermi velocity of the Dirac bands is also calculated for the BS structure under different strain values in order to assess the charge-carrier mobility. The band dispersions are anisotropic, and the following $k \bullet p$ model is used:

$$
\begin{gathered}
\qquad \begin{array}{c}
H(k)=A k_{x}+B k_{y}+\left(a k_{x}+c k_{y}\right) \sigma_{y} \\
+\left(b k_{x}+d k_{y}\right) \sigma_{x}+m \sigma_{z},
\end{array} \\
\text { Assume } k_{y}=0 \text {, then } \varepsilon_{ \pm}(k)=\left[A \pm \sqrt{a^{2}+b^{2}}\right] k_{x}, \\
\text { Assume } k_{x}=0 \text {, then } \varepsilon_{ \pm}(k)=\left[B \pm \sqrt{c^{2}+d^{2}}\right] k_{y} .
\end{gathered}
$$



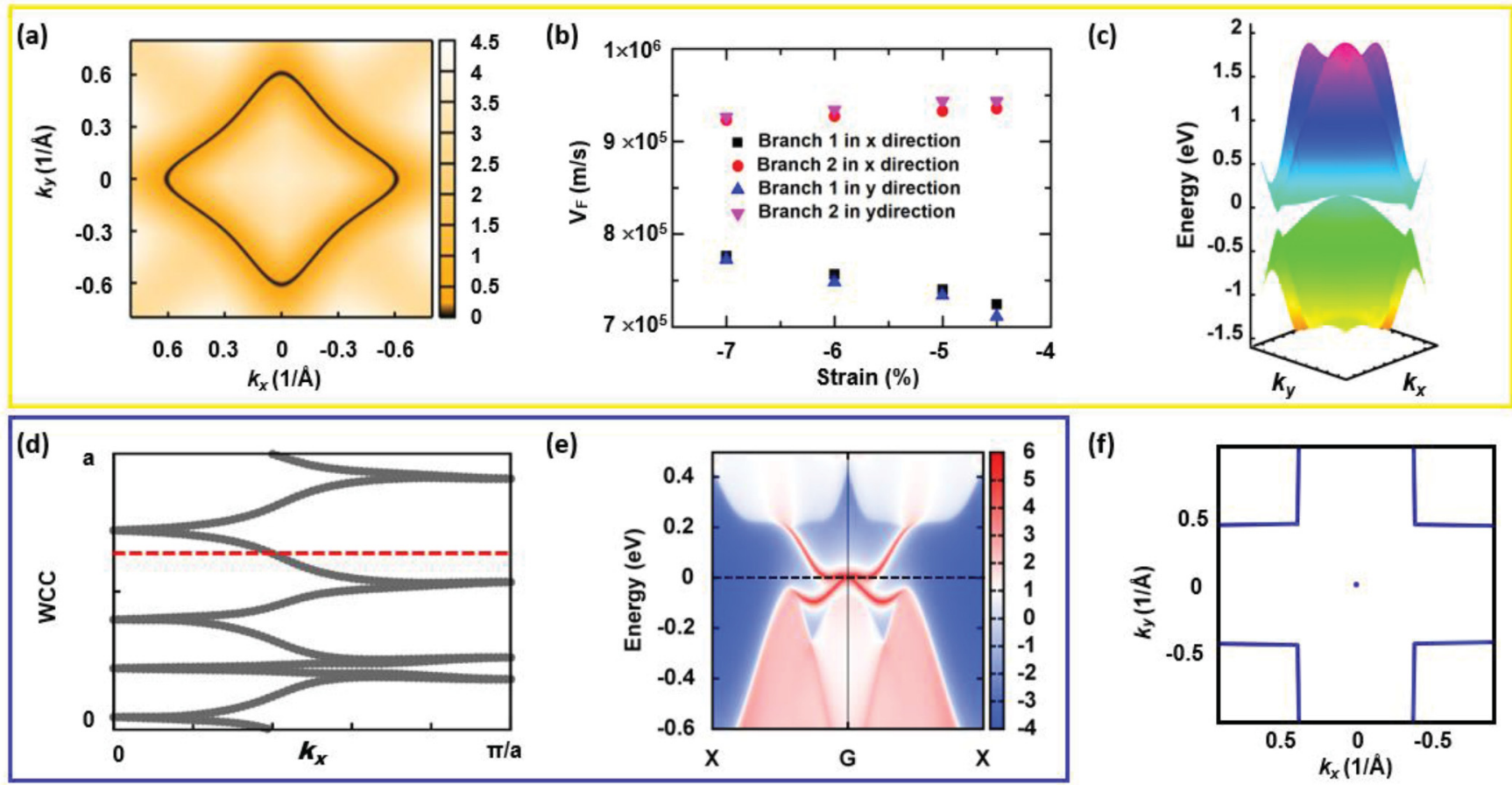

FIG. 4. Topological properties of the 2D tellurium system in phase I (a, b, c), phase II (d, e), and phase III (f). (a) A nodal line exists in the BS structure at $\Delta=-4.5 \%$ strain without SOC. (b) Fermi velocities of the BS structures under different stain. (c) Three-dimensional picture of a Dirac cone in the BS structure at $\Delta=-4.5 \%$ strain. (d) Evolution of the Wannier charge centers (WCC) along $k_{x}$ for the BR structure with a band gap of $0.24 \mathrm{eV}$. (e) Topological edge states connecting the conduction and valence bands of the BR structure with a band gap of $0.24 \mathrm{eV}$. (f) Schematic diagram showing the nodal line in the PS structure at $\Delta=13 \%$ strain without SOC (four right-angles shape). The blue point in the middle indicates two degenerate bands at the $\mathrm{G}$ point.

Because each single Dirac point deviates from the G point, it does not possess the fourfold rotation symmetry. Thus, the Fermi velocity of each Dirac point turns out to be directional and there are two different velocity branches along the $k_{x}$ and $k_{y}$ directions. Therefore, $\mathrm{A} \neq \mathrm{B}$, and $\sqrt{a^{2}+b^{2}} \neq \sqrt{c^{2}+d^{2}}$. These parameters with different strain are listed in Table S1 in the Supplemental Material [43]. To estimate the accuracy of the parameters, the parameters are fit to the first-principles energy bands of BS structures with various strain, as shown in Fig. S2 in the Supplemental Material [43]. The value of the larger velocity branches along the $k_{x}$ and $k_{y}$ directions slightly increase with the increase of strain, while the value of the smaller velocity branches along the $k_{x}$ and $k_{y}$ directions decrease with the increase of strain, as plotted in Fig. 4(b). The BS structure under strain of $\Delta=-4.5 \%$ has the highest Fermi velocity of $9.36 \times 10^{5} \mathrm{~m} / \mathrm{s}$ in the $k_{x}$ direction and $9.44 \times 10^{5} \mathrm{~m} / \mathrm{s}$ in the $k_{y}$ direction, which is comparable to that of graphene, while the lowest Fermi velocity is only about $75 \%$ of the highest one in each direction. The distortion of Dirac cones suggests high direction-dependent electrical conductivities. To give a visual description of the Dirac cones, a representative three-dimensional picture is shown in Fig. 4(c). Only one of the four Dirac cones is shown since four are related by symmetry.

Second, we further address the topological properties of phase II. The BR structure contains two band crossings along the $\mathrm{M}_{1}-\mathrm{G}$ direction without considering SOC. The non-SOC band structure is shown in Fig. 3(c). The valence-band max- imum and the conduction-band minimum are described by different IRs of the $\mathrm{C}_{2}$ point group. The IR of the band shown in red is A, while that of the band shown in black is B [see Fig. 3(c) inset] [41]. Thus, two Dirac points equivalent due to the twofold rotation symmetry are formed in the $\mathrm{M}_{1}-\mathrm{G}$ direction. In addition, it is worth noting that there exists a band gap along the $\mathrm{G}-\mathrm{M}_{2}$ direction, which can be understood via bond length analysis in the following way. Consider a structure characterized by $\Delta=4 \%$ strain. The lengths of short, long, and medium bonds are $2.82,3.47$, and $3.16 \AA$, respectively. The band inversion takes place at the $\mathrm{G}$ point, but the hybridization is mainly in the short bond along the G- $\mathrm{M}_{2}$ direction due to bond length difference, resulting in inequivalent band structures along the $\mathrm{G}-\mathrm{M}_{2}$ and $\mathrm{M}_{1}-\mathrm{G}$ directions. For this reason, one can see from Fig. 3(c) that the band gap opens only along the G- $\mathrm{M}_{2}$ direction, leaving the band crossing in the band inversion state along the $\mathrm{M}_{1}-\mathrm{G}$ direction.

When SOC is taken into account, the gap is opened at the Dirac point and the BR structure becomes a 2D TI, with the mechanism of this transformation being similar to that of the BS structure. The band-gap variation with strain is shown in Fig. 1(e). To verify the QSHE phase of the BR structure, we further evaluated the $Z_{2}$ invariant by tracing the evolution of the Wannier charge centers during an adiabatic pumping. The $Z_{2}$ invariant is identified by the odd number crossings of Wannier charge centers (black lines) of the reference line (red line). For example, the BR structure under $\Delta=4 \%$ strain has a $Z_{2}$ index (crossing number modulo 2) of 1, as shown in Fig. 4(d). 

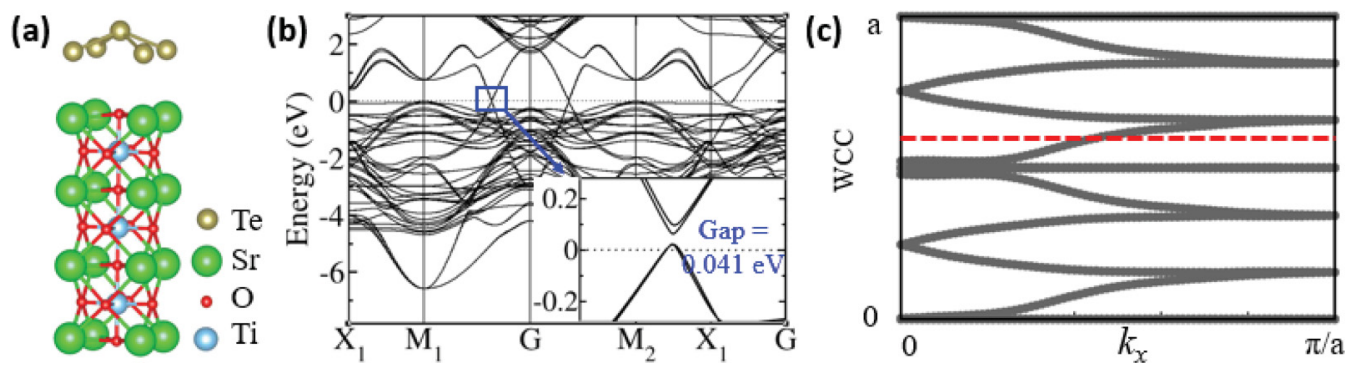

FIG. 5. Structure and electronic properties of the $\mathrm{BS} \mathrm{Te} / \mathrm{SrTiO}_{3}$ system. (a) Atomic structure of monolayer BS Te on the SrO-terminated $\mathrm{SrTiO}_{3}$ (001) surface. (b) Projected band structure of the $\mathrm{BS} \mathrm{Te} / \mathrm{SrTiO}_{3}$ system with $\mathrm{SOC}$ onto the monolayer tellurene. (c) Evolution of the Wannier charge centers (WCC) along $k_{x}$ for the $\mathrm{BS} \mathrm{Te} / \mathrm{SrTiO}_{3}$ system.

This indicates that the structure realizes the 2D QSHI phase, possessing the helical edge states connecting the conduction and valence bands. The nontrivial helical edge states can be obtained from the zigzag top edge (indicated by the transverse red dotted line) along the $x$ direction of the Te monolayer as shown in Fig. 2(b). And the surface states are calculated using the Green's-function method based on the tight-binding Hamiltonian as shown in Fig. 4(e). Clearly, the edge states (red lines) of the BR structure are present in the energy gap, which is different from the band structure of a trivial insulator. When a mild strain of $\Delta=4 \%$ is applied, the band gap reaches a maximum value of $0.24 \mathrm{eV}$, which makes applications in room-temperature devices feasible. The band gap is slightly enhanced when the calculations are performed using the hybrid functional HSE06. The QSHE phase is present in a broad range of strain from -4 to $7 \%$, indicating the potential applications under various mechanical environments. In addition, the band gap of the BR structure is always larger than $0.084 \mathrm{eV}$, which implies the robustness of the QSHE states. Hence, we argue that the helical edge states are protected from crystal structural deformation at ambient temperature.

Third, we address the topological properties of the overall metallic phase III. When not taking SOC into account, the structure exhibits a nodal line and a degeneracy. We first calculate the band structure without SOC and find there are three band crossings along the $\mathrm{X}_{1}-\mathrm{M}_{1}$ and $\mathrm{M}_{1}-\mathrm{G}$ directions [Fig. 3(e)]. Detailed wave-function analysis shows that the two band crossings, formed by bands shown in black and red, belong to different IRs of the $\mathrm{C}_{2 \mathrm{v}}$ point group [inset of Fig. 3(e)]. The IR of the band shown in red is $A_{1}$, while that of the band shown in black is $B_{1}$ [41]. Thus, in the non-SOC case, the band intersections can form a nodal line in the form of four right angles [illustrated in Fig. 4(f)], protected by the PT symmetry. In addition, it is worth noting that along the $\mathrm{M}_{1}-\mathrm{G}$ direction the bands indicated in green and red are degenerate at the $G$ point. We indicate this degeneracy by the blue point in the middle of the schematic diagram [Fig. 4(f)]. Furthermore, the other band crossing formed by the bands shown in black and green (along the $\mathrm{M}_{1}-\mathrm{G}$ direction) is also described by different group IRs. This means that this band crossing could lead to another nodal line, but its energy is much lower than the Fermi level. When SOC is included, the band gap is opened along the nodal line near the Fermi level. A large band gap is opened along the $X_{1}-M_{1}$ direction, while a smaller band gap of $0.06 \mathrm{eV}$ is seen along the $\mathrm{M}_{1}-\mathrm{G}$ direction [inset of Fig. 3(f)].

\section{Tellurene on $\mathrm{SrTiO}_{3}$}

Finally, we would like to discuss the possibility of experimental synthesis of the BS structure in phase I. We suggest the insulating $\mathrm{SrTiO}_{3}$ substrate as a possible candidate to stabilize the strained structure, with the reasons being that (1) $\mathrm{SrTiO}_{3}$ has a large experimental energy gap of $3.2 \mathrm{eV}$ [44] $(1.84 \mathrm{eV}$ as theoretical value [45]), which can well preserve the topological properties of tellurium around the Fermi level, and (2) the small lattice mismatch $(<1 \%)$ between the lattice constant of $\mathrm{SrTiO}_{3}(a=3.91 \AA)$ [46] and that of BS Te $(a \sim 3.94 \AA)$ may allow growing monolayer tellurene. The calculation of the $\mathrm{BSTe} / \mathrm{SrTiO}_{3}$ model is displayed in Fig. 5(a). The band structure shows that four 2D Dirac cones in the BS phase I remain and a small SOC gap of $0.041 \mathrm{eV}$ is opened due to the substrate effect. However, comparing the SOC band structure in Fig. 3(b) with that in Fig. 5(b), we can see that the Dirac bands along the $\mathrm{M}_{1}-\mathrm{G}$ and $\mathrm{G}-\mathrm{M}_{2}$ directions in the $\mathrm{BSTe} / \mathrm{SrTiO}_{3}$ system are not degenerate any more since inversion symmetry of the BS structure is broken by the substrate. In addition, to further verify that the topological phase of BS Te is not affected, we calculated its $Z_{2}$ invariant using the same method as described above [47]. The result shows that the $Z_{2}$ index is 1 , confirming the topological properties of Dirac cones, around which electrons can move with high mobility. The calculated Fermi velocity of the $\mathrm{BSTe} / \mathrm{SrTiO}_{3}$ system is direction dependent. The BS Te $/ \mathrm{SrTiO}_{3}$ system shows the largest Fermi velocity of $8.97 \times 10^{5} \mathrm{~m} / \mathrm{s}$ along the $k_{x}$ direction and $8.99 \times 10^{5} \mathrm{~m} / \mathrm{s}$ along the $k_{y}$ direction [related parameters in Eq. (1) are listed in Table S1 in the Supplemental Material and parameter fitting bands are shown in Fig. S2 in the Supplemental Material [43]], which are slightly smaller than those for the corresponding 2D BS structure. These results reveal that the presence of $\mathrm{SrTiO}_{3}$ substrate does not affect the Dirac fermion band dispersion of the BS structure. In addition, for the BR Te monolayer structure, studies of phonon dispersions and molecular dynamics simulations confirm that its structure is stable [37]. We also note that the PS Te can be grown on the Au (100) surface even when the lattice constant is as large as $7 \AA$, as indicated by in situ atomic force microscopy [48]. We further calculate the cohesive energy of the Te structure in each phase. The calculated cohesive energies are $2.49,2.52$, and $2.29 \mathrm{eV} /$ atom for the BS structure $(\Delta=-4.5 \%)$, BR structure $(\Delta=4 \%)$, and PS structure $(\Delta=13 \%)$, respectively. These values are comparable to the cohesive energy of $\beta$-Te $(2.56 \mathrm{eV} /$ atom $)$ which has been successfully fabricated in experiment [35]. 
This also indicates the potential of experimental preparation of the 2D tellurium system.

\section{CONCLUSIONS}

In summary, crystalline structures and electronic properties of a monolayer $2 \mathrm{D}$ square tellurium have been investigated using first-principles calculations. The buckled 2D tellurium system shows high tunability by strain, which manifests in topological phase transitions and has a strong effect on the electronic structure. Three structural phases are identified in the $2 \mathrm{D}$ Te system - the buckled square phase I, the buckled rectangular phase II, and the planar square phase III. The energy difference between these phases is relatively small, which favors the phase transitions. In the buckled square phase I under $\Delta=-4.5 \%$ strain, there are four Dirac points with holes as charge carriers and a large Fermi velocity of $9.44 \times 10^{5} \mathrm{~m} / \mathrm{s}$. Upon decreasing strain, the gap is opened at the Dirac points and its value is much larger than that of graphene. The buckled rectangular phase II shows the QSHE phase in a wide strain range from -4 to $7 \%$. The QSHE is confirmed by evaluation of the $Z_{2}$ invariant using the hybrid Wannier function technique. The maximum direct band gap of the buckled rectangular structure is $0.24 \mathrm{eV}$ under a strain of $\Delta=4 \%$, which implies robustness of the QSHE required for room-temperature applications. When strain $\Delta$ is greater than $8 \%$, the planar square phase III is realized. Without considering SOC, nodal-line degeneracies are present in the buckled square and planar square structures. The results also indicate that the topological properties can be controlled by structural changes rather than SOC as it was suggested in our previous papers [22-24]. The versatile electronic properties of the 2D square tellurium could be utilized in numerous prospective applications.

\section{ACKNOWLEDGMENTS}

This work was supported by the National Natural Science Foundation of China (Grants No. 11504051 and No. 21703248), the Strategic Priority Research Program of the Chinese Academy of Sciences (Grant No. XDB20000000), and Natural Science Foundation of Fujian Province of China (Grant No. 2018J06001). Z.X.G. is supported by an Engineering and Physical Sciences Research Council grant (Grant No. EP/N032888/1), Q.W. and O.V.Y. are supported by National Centre of Competence in Research Marvel.
[1] K. S. Novoselov, A. K. Geim, S. V. Morozov, D. Jiang, M. I. Katsnelson, I. V. Grigorieva, S. V. Dubonos, and A. A. Firsov, Nature (London) 438, 197 (2005).

[2] A. H. C. Neto, F. Guinea, N. M. R. Peres, and K. S. N. A. Geim, Rev. Mod. Phys. 81, 109 (2009).

[3] F. Zhu, W. Chen, Y. Xu, C. Gao, D. Guan, C. Liu, D. Qian, S. Zhang, and J. Jia, Nat. Mater. 14, 1020 (2015).

[4] A. Molle, J. Goldberger, M. Houssa, Y. Xu, S. Zhang, and D. Akinwande, Nat. Mater. 16, 163 (2017).

[5] G. Fiori, F. Bonaccorso, G. Iannaccone, T. Palacios, D. Neumaier, A. Seabaugh, S. K. Banerjee, and L. Colombo, Nat. Nanotech. 9, 768 (2014).

[6] D. Akinwande, L. Tao, Q. Yu, X. Lou, P. Peng, and D. Kuzum, IEEE Nanotechnol. Mag. 9, 6 (2015).

[7] A. C. Ferrari, F. Bonaccorso, V. Fal'Ko, K. S. Novoselov, S. Roche, P. Boggild, S. Borini, F. H. L. Koppens, V. Palermo, N. Pugno, J. A. Garrido, R. Sordan, A. Bianco, L. Ballerini, M. Prato, E. Lidorikis, J. Kivioja, C. Marinelli, T. Ryhanen, A. Morpurgo, J. N. Coleman, V. Nicolosi, L. Colombo, A. Fert, M. Garcia-Hernandez, A. Bachtold, G. F. Schneider, F. Guinea, C. Dekker, M. Barbone, Z. Sun, C. Galiotis, A. N. Grigorenko, G. Konstantatos, A. Kis, M. Katsnelson, L. Vandersypen, A. Loiseau, V. Morandi, D. Neumaier, E. Treossi, V. Pellegrini, M. Polini, A. Tredicucci, G. M. Williams, B. H. Hong, J. H. Ahn, J. M. Kim, H. Zirath, B. J. van Wees, H. van der Zant, L. Occhipinti, A. Di Matteo, I. A. Kinloch, T. Seyller, E. Quesnel, X. Feng, K. Teo, N. Rupesinghe, P. Hakonen, S. R. T. Neil, Q. Tannock, T. Lofwander, and J. Kinaret, Nanoscale 7, 4598 (2015).

[8] F. Yang, L. Miao, Z. F. Wang, M. Y. Yao, F. Zhu, Y. R. Song, M. X. Wang, J. P. Xu, A. V. Fedorov, Z. Sun, G. B. Zhang, C. Liu, F. Liu, D. Qian, C. L. Gao, and J. F. Jia, Phys. Rev. Lett. 109, 016801 (2012).

[9] Z. Wang, X. Zhou, X. Zhang, Q. Zhu, H. Dong, M. Zhao, and A. R. Oganov, Nano Lett. 15, 6182 (2015).
[10] L. Kou, Y. Ma, Z. Sun, T. Heine, and C. Chen, J. Phys. Chem. Lett. 8, 1905 (2017).

[11] S. Cahangirov, M. Topsakal, E. Akturk, H. Sahin, and S. Ciraci, Phys. Rev. Lett. 102, 236804 (2009).

[12] Y. Xu, B. Yan, H. J. Zhang, J. Wang, G. Xu, P. Tang, W. Duan, and S. C. Zhang, Phys. Rev. Lett. 111, 136804 (2013).

[13] A. J. Mannix, X. F. Zhou, B. Kiraly, J. D. Wood, D. Alducin, B. D. Myers, X. Liu, B. L. Fisher, U. Santiago, J. R. Guest, M. J. Yacaman, A. Ponce, A. R. Oganov, M. C. Hersam, and N. P. Guisinger, Science 350, 1513 (2015).

[14] S. Das, W. Zhang, M. Demarteau, A. Hoffmann, M. Dubey, and A. Roelofs, Nano Lett. 14, 5733 (2014).

[15] K. Asano and C. Hotta, Phys. Rev. B 83, 245125 (2011).

[16] J. Wang, S. Deng, Z. Liu, and Z. Liu, Natl. Sci. Rev. 2, 22 (2015).

[17] S. M. Nie, Z. Song, H. Weng, and Z. Fang, Phys. Rev. B 91, 235434 (2015).

[18] Y. Ma, L. Kou, X. Li, Y. Dai, and T. Heine, NPG Asia Mater. 8, e264 (2016).

[19] X. F. Zhou, X. Dong, A. R. Oganov, Q. Zhu, Y. Tian, and H. T. Wang, Phys. Rev. Lett. 112, 085502 (2014).

[20] F. Bonaccorso, Z. Sun, T. Hasan, and A. C. Ferrari, Nat. Photon. 4, 611 (2010).

[21] H. Zhang, C. Liu, X. Qi, X. Dai, Z. Fang, and S. Zhang, Nat. Phys. 5, 438 (2009).

[22] W. Zhang, R. Yu, W. Feng, Y. Yao, H. Weng, X. Dai, and Z. Fang, Phys. Rev. Lett. 106, 156808 (2011).

[23] W. Zhang, R. Yu, H. Zhang, X. Dai, and Z. Fang, New J. Phys. 12, 065013 (2010).

[24] R. Yu, W. Zhang, H. J. Zhang, S. C. Zhang, X. Dai, and Z. Fang, Science 329, 61 (2010).

[25] G. Kresse and J. Furthmüller, Comp. Mater. Sci. 6, 15 (1996).

[26] G. Kresse and J. Furthmüller, Phys. Rev. B 54, 11169 (1996). 
[27] J. P. Perdew, K. Burke, and M. Ernzerhof, Phys. Rev. Lett. 77, 3865 (1996).

[28] J. Heyd, G. E. Scuseria, and M. Ernzerhof, J. Chem. Phys. 118, 8207 (2003).

[29] J. Heyd, G. E. Scuseria, and M. Ernzerhof, J. Chem. Phys. 124, 219906 (2006).

[30] N. Marzari and D. Vanderbilt, Phys. Rev. B 56, 12847 (1997).

[31] I. Souza, N. Marzari, and D. Vanderbilt, Phys. Rev. B 65, 035109 (2001).

[32] M. Dion, H. Rydberg, E. Schröder, D. C. Langreth, and B. I. Lundqvist, Phys. Rev. Lett. 92, 246401 (2004).

[33] T. Thonhauser, V. R. Cooper, S. Li, A. Puzder, P. Hyldgaard, and D. C. Langreth, Phys. Rev. B 76, 125112 (2007).

[34] X. Luo, M. B. Sullivan, and S. Y. Quek, Phys. Rev. B 86, 18411 (2012).

[35] Z. Zhu, X. Cai, S. Yi, J. Chen, Y. Dai, C. Niu, Z. Guo, M. Xie, F. Liu, J.-H. Cho, Y. Jia, and Z. Zhang, Phys. Rev. Lett. 119, 106101 (2017).

[36] Y. Wang, C. Xiao, M. Chen, C. Hua, J. Zou, C. Wu, J. Jiang, S. A. Yang, Y. Lu, and W. Ji, Mater. Horiz. 5, 521 (2018).

[37] L. Xian, A. P. Paz, E. Bianco, P. M. Ajayan, and A. Rubio, 2D Mater. 4, 041003 (2017).
[38] Z. Song, S. M. Nie, H. Weng, and Z. Fang, arXiv:1508.05220v1 (2015).

[39] W. Luo and H. Xiang, Nano Lett. 15, 3230 (2015).

[40] G. C. Vezzoli, Z. Kristallogr. Bd. 134, 305 (1971).

[41] S. Altmann and P. Herzig, Point-Group Theory Tables (Clarendon, Oxford, 1994).

[42] Y. Yao, F. Ye, X. L. Qi, S. C. Zhang, and Z. Fang, Phys. Rev. B 75, 041401 (2007).

[43] See Supplemental Material at http://link.aps.org/supplemental/ 10.1103/PhysRevB.98.115411 for the SOC energy bands for structures with different strain in BS phase I, and parameters used in Eq. (1) for BS structures with different strain and $\mathrm{BS} \mathrm{Te} / \mathrm{SrTiO}_{3}$.

[44] M. Cardona, Phys. Rev. 140, A651 (1965).

[45] G. Fabricius, E. L. Peltzer y Blanca, C. O. Rodriguez, A. P. Ayala, P. de la Presa, and A. Lopez García, Phys. Rev. B 55, 164 (1997).

[46] Z. Wang, R. Sun, C. Chen, M. Saito, S. Tsukimoto, and Y. Ikuhara, J. Mater. Sci. 47, 5148 (2012).

[47] Q. Wu, S. Zhang, H.-F. Song, M. Troyer, and A. A. Soluyanov, Comput. Phys. Commun. 224, 405 (2018).

[48] N. Ikemiya, D. Iwai, K. Yamada, R. Vidu, and S. Hara, Sur. Sci. 369, 199 (1996). 